\title{
Using thinking aloud to enhance poor readers' vocabulary knowledge

\author{
and comprehension
}

\author{
Wen-qi Xiao \\ B511-2, Kunluan Building, International College, Xiamen University (Xiang-an Campus), Xiang-an south road, Xiang-an District, Xiamen, Fujian, China
}

Key words: thinking aloud, poor readers, vocabulary knowledge, reading comprehension

\begin{abstract}
English reading proficiency is regarded as one of the major abilities of English language learning. However, poor readers who lack vocabulary knowledge and have difficulty in monitoring themselves in reading process struggle a lot in L2 reading. The aim of the study attempts to discuss poor readers reading problems and to explore how thinking aloud can enhance vocabulary knowledge and comprehension. Through literature research, the study finds that thinking aloud can be applied to help poor readers' problems and also assess readers' strengths and weaknesses. The revelation from thinking aloud can be further employed by teachers to adjust instruction on the basis of students' needs.
\end{abstract}

\section{Introduction}

It is hard for English language teachers to explicitly find out the comprehending process in students' mind, as students approach to L2 reading. However, the significance of reading in L2 learning and the large share it takes up in English examinations force some language teachers to adopt drillings for improving their reading performance. This approach aiming at consolidating certain reading skills may be effective for competent readers, but this practice will probably be ineffective for poor readers who are unaware of using strategies and have difficulty in monitoring themselves in reading process. Thinking aloud emphasizing on verbalizing the thoughts and strategies during the reading process [1] is a protocol for poor readers to construct reading strategies and enhance their vocabularies in reading comprehension, and at the same time this process will be reference for teachers to identify poor readers' problems and tailor their instruction in turn.

Recent years of second language reading research has focused on reading strategies that readers adopt to facilitate or construct comprehension [2, 3, 4]. It is suggested that proficient readers use a variety of strategies to generate meaning, while poor readers lack vocabularies and strategies to approach reading and often fail to monitor their reading process $[1,5]$. How to explicitly examine the thinking process when poor readers read become a issue to be identified first. Think-aloud protocol operating as one of the important data source for cognitive process [1] asks readers to verbalize or say aloud what comes from their minds when they read $[1,6,7]$. While poor readers use this method, instructors can notice the cognitive process and exact problems causing comprehension failure, and demonstrate appropriate thinking process for poor readers to model and learn. The demonstration and imitation will help poor readers to construct metacognitive awareness and reading strategies [6]. Recent research has also put emphasis on using the revealing from think-aloud as teaching references for teachers to modify their instructions [6]. In this study, I will first describe poor readers' problems in reading. Then I will analyze how thinking aloud is 
applicable for poor readers to improve their vocabularies and comprehension. Finally, implications for teaching poor readers' will be discussed.

\section{The poor readers' problems}

\section{Inadequate vocabulary knowledge}

Limited vocabulary size. The reciprocal relationship between vocabulary and reading comprehension indicates that vocabulary is an important factor in facilitating comprehension, and reading experience in turn can develop vocabulary knowledge [8]. As for the poor readers who have small vocabulary size, it is difficult for them to comprehend the text at first place, and the chance for them to obtain extra word knowledge is much lower. In compared to good readers, poor readers read less and less, consequently, drifting away from the peers or even giving up reading. From the language threshold theory perspective, it suggests that L2 readers are supposed to obtain sufficient L2 linguistic knowledge, for instance words, syntax, so that they can effectively transfer their L1reading skills in L2 reading. Although this hypothesis is criticized by researchers, it is worth noting "a variable amount of linguistic knowledge is needed to read a specific text..." [9, P.50]. Increasing the vocabulary size of the poor readers is a crucial building block for them to catch up and become better readers. Meanwhile, how to teach vocabulary for poor readers is another issue to be addressed. Nation [10] has stated that vocabulary knowledge includes not only knowing the word form and its meaning, but also its syntactic rules and when, and where to use it [11]. This attaches the importance of teaching vocabulary in meaningful and specific contexts instead of mechanic memorization.

Lacking morphological knowledge. Researches have found that an understanding of morphology is linked with reading comprehension $[12,13]$ and there appears to be a reciprocal relationship between morphology and vocabulary [13]. Large vocabulary leads to a better understanding of morphology knowledge, and understanding morphology can broaden students' vocabularies. The relationship implies that teaching morphology can facilitate students' comprehension as well.

For instance, poor readers may recognize the word 'tour', but may fail to infer the meaning of the word 'tourist', which is combined by the root word: tour and the suffix morpheme: -ist. Therefore, explicit instruction on morphological knowledge should be integrated in context-rich vocabulary teaching, and teaching students to utilize the knowledge as a cognitive strategy in reading.

\section{Lower degree of metacognitive awareness}

Metacognition or metacognitive awareness refers to the knowledge of understanding and controlling one's cognitive behaviors [14]. Generally, there are two dimensions of metacognitive ability: knowledge of cognition and knowledge of regulation. Knowledge of cognition enables the learner to be aware of the learning style or characteristics of himself or herself, the structures of different types of texts and the related strategies to cope with the text demands. In other words, knowledge of cognition reflects leaners belief that "unlike other people, they should use strategy A rather than B in task X rather than task $Y$ to achieve the goal of learning." [15, P.4] Studies in L2 reading have proved that effective readers obtain a larger variety of strategies, and are able to select and allocate them in a more effective way $[15,16]$. 
Knowledge of regulation performs the role of managing the cognitive process. It comprises three skills (1) planning, i.e. understanding the purpose or goal of reading, selecting appropriate strategies, and making predictions before reading, (2) monitoring, i.e. on-going regulation of one's comprehension during reading (3) evaluating, i.e. appraising or re-evaluating one's outcomes or ability in achieving the goal. Reading research has also revealed that low-proficiency readers tend to rely only on bottom-up strategies (e.g. word by word recognition) in reading, while advanced learner are more likely to obtain a global planning (e.g. structure of the text) for reading $[15,17]$.

The students who are struggling with English reading has similar problems due to their lower degree of metacognitive awareness. With little understanding of text structure, they seldom hold goals and exceptions for the reading texts, and tend to read word by word. Once they fail to retrieve the word meaning from their memory, they may easily give up the reading. This also reflects that they lack word awareness. In fact, reading comprehension does not mean that one has to recognize every word, and effective readers often infer the meanings by using context clues or morphological analysis.

\section{Application of think-aloud}

Thinking aloud and vocabulary learning. In terms of vocabulary teaching, both direct and indirect methods should be used. Direct teaching involves giving examples or using synonyms. Indirect teaching includes "using strategies such as context clues, or morphemic analysis, so that the students can determine the meaning of a word on their own." [18, p.143] Even though some vocabularies that are key to comprehension require instruction before reading the text, teachers are not suggested to explicitly teach all the new words to students in the pre-reading section. For the words whose meaning can be deduced from textual content, or from morphological knowledge, teachers should use think-aloud as a protocol to model the process of inferring words meaning. Since poor readers are weak at morphological knowledge and have lower awareness of using strategies for word recognition, it is more necessary for teachers to integrate morphological knowledge with vocabulary instruction, and then model the process in the while-reading part. Spencer [19]] has pointed out that adopting the thinking aloud to support students' growth in metacognition, so that they can monitor their word knowledge.

Thinking aloud and metacognitive awareness. When the think-aloud is adopted as a reading approach, readers are supposed to stop periodically, and check the comprehension process by verbally relating to what strategies are used. The think-aloud approach itself is a comprehension-monitoring tool, and moreover it presents the access and use of various skills. Considering this, thinking aloud is closely related with metacognition. As mentioned above, metacognition contains knowledge of cognition and knowledge of regulation. Through applying the think-aloud to reading, the method not only enables readers to know when and how to use reading strategies (knowledge of cognition), but also acts as an on-going regulation (knowledge of regulation). This is evidenced by research study. A comparison has been made between children who have attended a think-aloud program and those who have not. The result shows that the think-aloud group are able to reflect on whether or not comprehension is occurring, and demonstrate various strategies such as self-questioning, verifying, whereas the latter group failed to do so [7]. 
From the above analysis, we can see that thinking aloud as an instruction approach can be adopted to address the poor readers' problems. In the pre-reading session, explicit instruction on the meanings of core vocabularies and morphological knowledge should be taught to increase poor readers' vocabulary knowledge as well as vocabulary learning strategies. During the while-reading process, it is suggested to use thinking aloud as a tool to facilitate comprehension. Teachers should demonstrate the think-aloud process by asking self-directed questions such as : What's my plan for the reading? What does that mean? Dose it make sense? to present the cognitive process and reading strategies, and students model the procedure and will also fill the self-evaluation sheets to be aware of the strategies they use. When applying the think-aloud technique, special emphasis should be made on using morphological knowledge and contextual clues. In terms of metacognition training, focus should be put on reading purpose, textual organization, strategy using and on-going monitoring. As students use think aloud, their weakness and strengths will be revealed as well and instructions can be modified to specifically address their problems and needs.

\section{Conclusion}

Poor readers are often described as passive, or demotivated in reading. However, as teachers we should not give up the group of readers who in fact need more support and help to build confidence in reading and catch up with the peers. Therefore, identifying their specific deficiencies is the initial step, so that we can come up with corresponding and effective methods to solve their problems.

Inadequate vocabulary knowledge and lower degree of metacognition are the major problems that poor readers' comprehension in English reading. Thinking aloud as a protocol to verbalize thoughts can address the problems. Teachers are supposed to demonstrate the thinking aloud procedure and ask students to model and learn. During the process, self-directed questions are frequently asked by readers to check the on-going reading. Think-aloud training should focus on utilizing morphological knowledge and contextual clues to infer meaning and learn new words. At the same time, reading purpose, textual organization, strategy using should be analyzed to enhance metacognition awareness. However, vocabulary knowledge and reading comprehension can not be improved in a short period of time. Systematic, and consistent training by using and practicing thinking aloud is needed to help poor readers become confident and strategic readers. Only through consistent training of thinking aloud, poor readers can become better readers and hold interest in L2 reading.

\section{References}

[1] S. E. Wade, Using think alouds to access comprehension. The Reading Teacher. 43 (1990), 442-451.

[2] M. Ghavamnia, S. Ketabi, M. Tavakoli, L2 Reading strategies used by Iranian EFL learners: A think-aloud study, Reading Psychology. 34 (2013), 355-378.

[3] N. J. Andreson, Individual differences in strategy use in second language reading and testing, Modern Language Journal. 75 (1991), 460-472.

[4] P. L. Carrell, SLA and classroom instruction, Reading Annual Review of Applied Linguistics. 9 (1988), 223-242.

[5] S. G. Paris, M. Y. Lipson, K. K. Wixson, Becoming a strategic reader, Comtemporary Educational Psychology. 8 (1983), 293-316.

[6] L. Oster, Using the think-aloud for reading instruction, The Reading Teacher. 55 (2001), 64-69.

[7] J. F. Baumann, L. A. Jones, N. S. Kessell, Using think alouds to enhance children's 
comprehension monitoring, The Reading Teacher. 47 (1993), 184-194.

[8] P. G. Aaron, R. M Joshi, Reading Problems Consultation and Remediation, The Guilford Press, New York, 1992.

[9] W. Grabe, F. L. Stoller, Teaching and researching reading, Longman, Harlow, 2002.

[10] I. S. P. Nation, Learning vocabulary in another language, Cambridge University Press, Cambridge, 2001.

[11]J. S. Hedgcock, D.R. Ferris, Teaching readers of English, Routledge, New York, 2009.

[12] J. F. Carlisle, Awareness of structure and meaning of morphological complex words: Impact on reading, Reading and Writing: An Interdisciplinary Journal. 12 (2000), 169-190.

[13] M. J. Kieffer, N. K. Lesaux, Breaking Down Words to Build Meaning: Morphology,Vocabulary, and Reading Comprehension in the Urban Classroom. The Reading Teacher. 6 (2007), 134-144.

[14] T. Hudson, Teaching second language reading, Oxford UniversityPress, Oxford, 2007.

[15] L. J. Zhang, Awareness in Reading: EFL Students' Metacognitive Knowledge of Reading Strategies in an Acquisition-poor Environment, Language Awareness. 10 (2001), 268-288.

[16] R. E. Reynolds, Selective attention and prose learning: Theoretical and empirical research, Educational Psychology Review. 4 (1992), 345-391.

[17] P. L. Carrell, Metacognition awareness and second language reading, Modern Language Journal. 73 (1989), 121-134.

[18] W. D. Bursuck, B. M. Damer, Teaching reading to students who are at risk or have disputabilities, Pearson, New Jersey, 2007.

[19] B. H. Spencer, A. M. Guillaume, Integrating curriculum through the learning cycle: Content-based reading and vocabulary instruction, The Reading Teacher. 60 (2006), 206-220. 